\title{
Effect of high-fat-fructose diet on synaptic plasticity in hippocampus and lipid profile of blood serum of rat: pharmacological possibilities of affecting risk factors
}

Original research article/Review

Micháliková D ${ }^{1,2 \boxminus}$, Tyukos Kaprinay B. ${ }^{1,2}$, Lipták B. ${ }^{1,2}$, Švík K. ${ }^{1}$, Slovák L. ${ }^{1}$, Sotníková R. ${ }^{1}$, Bezek Š. ${ }^{1}$, Gáspárová Z.

${ }^{1}$ Slovak Academy of Sciences, Centre of Experimental Medicine, Institute of Experimental Pharmacology and Toxicology, Bratislava, Slovak Republic ${ }^{2}$ Comenius University in Bratislava, Jessenius Faculty of Medicine in Martin, Martin, Slovak Republic

Received 22 June, 2016, accepted 19 July, 2016

Abstract The aim of this study was to determine pharmacological possibilities of influencing the risk factors of metabolic syndrome (MetS). Hypertriacylglycerolemic (HTG) rats fed with high-fat-fructose diet (HFFD) were used as a model of the MetS. Wistar rats fed with standard diet were used as negative control group. HTG rats fed with HFFD for 8 weeks were used as positive control group. The effects of atorvastatin and SMe1EC2 were tested. The compounds were administered to the HTG rats after 5 weeks of HFFD, once a day for 3 weeks. After 8 weeks, the blood serum lipid profile and electrophysiology of neurotransmission in hippocampal sections were evaluated in vitro. SMe1EC2 and atorvastatin had a significant effect on total cholesterol (TC) and low-density lipoprotein cholesterol (LDL-cholesterol) and atorvastatin had a significant effect on triacylglycerols (TGs). SMe1EC2 improved the long-term potentiation (LTP) course in the hippocampus.

Keywords metabolic syndrome-high-fat-fructose diet-hippocampus-cholesterol-triacylglycerols-rat

\section{INTRODUCTION}

Metabolic syndrome (MetS) is a cluster of risk factors, including visceral obesity, insulin resistance, dyslipidemia, and hypertension, that, in conjunct, can lead to the development of type 2 diabetes mellitus and cardiovascular diseases (O'Neil and O'Driscoll, 2014). The risk of developing MetS depends on both genetic and life-style factors. The main risk factor for the development of MetS is physical inactivity and food consumption based on large amounts of fat and sugars (Janczura et al., 2015). Some risk factors for MetS are associated with the development of dementia and exert a negative influence on cognitive functions. It is not clear whether MetS as a whole syndrome or its individual components are more likely to contribute to the decline in cognition and development of dementia (Launer et al., 1995; Qiu et al., 2005). MetS is associated with impaired microstructural integrity of white brain mass in the parahippocampal gyrus and the hippocampus. In addition, MetS may lead to reduced hippocampal volume and increased cerebrospinal fluid volume (Yau et al., 2012; Alfaro et al., 2016). In rats, changes in hippocampal neuronal plasticity were also confirmed as related to MetS (Treviño et al., 2017). There is a great number of potential links between MetS and cognitive impairment. These include inflammation in the nervous system, oxidative stress, abnormal lipid metabolism in the brain, and impaired vascular reactivity. Insulin resistance can affect synaptic plasticity through damage to insulin-sensitive processes, which are responsible for neuronal survival, learning, and memory (Liu et al., 2015; Yates et al., 2012). Treatment that exists in this area is focused on influences of individual components of MetS. In MetS, several components are presented at the same time. Their treatment individually represents an increase in the number of undesirable effects of all concomitant medications. The aim of our work was to find whether the tested substances would affect several comorbidities associated with MetS. 


\section{METHODS}

Male hypertriacylglycerolemic (HTG) rats $(n=40)$ and Wistar rats $(n=10)$ were divided into 5 groups: Wistar rats, the negative control group fed with standard diet; HTG rats, the positive control group fed with high-fat-fructose diet (HFFD); the HTG group fed for 8 weeks with HFFD and treated from the 6 th to 8 th week with atorvastatin $(25 \mathrm{mg} /$ $\mathrm{kg}$ ); the HTG group fed for 8 weeks with HFFD and treated from the 6th to 8th week with SMe1EC2 with lower dosage $(0.5 \mathrm{mg} / \mathrm{kg})$; and the HTG group fed for 8 weeks with HFFD and treated from the 6th to 8th week with SMe1EC2 with higher dosage $(25 \mathrm{mg} / \mathrm{kg})$. The substances were administered intraperitoneally once a day at 9:00 a.m. for 3 weeks. Methylcellulose (vehiculum) was administered to both the control groups of rats. Standard rodent diet was produced by the certified producer of pellets at the Department of Toxicology and Breeding of Laboratory Animals, Institute of Experimental Pharmacology and Toxicology, Slovak Academy of Sciences, Dobra Voda, Slovakia, which is registered under number a SK 100089, the code 6147. The composition of the standard diet is given as follows: wheat, processed animal protein, oats, barley-corn, extruded lucerne, oil soybean meat, wheat bran, wheat germs, mineral mix, vegetable oil, sodium chloride. Vitamins added per $1 \mathrm{~kg}$ of the standard diet included the following: 20,000 IU of E672 vitamin A, 2,000 IU of E671 vitamin D3, and $70 \mathrm{mg}$ of vitamin E; aminoacids added per $1 \mathrm{~kg}$ of the standard diet included the following: $1.2 \mathrm{~g}$ of DL-methionine and $0.8 \mathrm{~g}$ of L-lysine. Analytical components used in the standard diet included the following: $19.10 \%$ of nitrogen substances, 3.60\% of fiber, $5.10 \%$ of oil and fat, $5.85 \%$ of ash, and $9.10 \%$ of humidity. High-fat diet (HFD) was enriched with about $1 \%$ of cholesterol, $7.5 \%$ of lard, and $10 \%$ of fructose. Rats had free access to water and food pellets and were kept on a 12-h/12-h light/dark cycle. All procedures involving the animals were performed in compliance with the Principles of Laboratory Animal Care issued by the Ethical Committee of the Institute of Experimental Pharmacology and Toxicology, Slovak Academy of Sciences and by the State Veterinary and Food Administration of Slovakia. The rats were decapitated under short ether anesthesia. Biochemical determination of lipid profile was performed on blood serum sample of each rat. Electrophysiological measurement of neurotransmission was performed on slices of left hippocampus of each rat. Lipid profile was determined by enzymatic photometric kit assay in vitro. Total cholesterol (TC), low-density lipoprotein cholesterol (LDL-cholesterol), high-density lipoprotein cholesterol (HDL-cholesterol), and triacylglycerols (TG) were examined. Neurotransmission was determined by recording and digitizing electrically induced responses of hippocampus. We used artificial cerebrospinal fluid (ACSF) composed of $124 \mathrm{mmol} / \mathrm{l}$ of $\mathrm{NaCl}, 3.3 \mathrm{mmol} / \mathrm{l}$ of $\mathrm{KCl}, 1.25$ $\mathrm{mmol} / \mathrm{l}$ of $\mathrm{KH}_{2} \mathrm{PO}_{4^{\prime}} 2.4 \mathrm{mmol} / \mathrm{l}$ of $\mathrm{MgSO}_{4^{\prime}} 2.5 \mathrm{mmol} / \mathrm{l}$ of $\mathrm{CaCl}_{2}, 26 \mathrm{mmol} / \mathrm{l}$ of $\mathrm{NaHCO}_{3}$, and $10 \mathrm{mmol} / \mathrm{l}$ of glucose and saturated with $95 \% \mathrm{O}_{2}+5 \% \mathrm{CO}_{2}$, at $\mathrm{pH}$ 7.4. Synaptic plasticity was evoked using high-frequency stimulation (HFS) of Schaffer collaterals. Hippocampal slices (400$\mu \mathrm{m}$ thick) were stimulated by bipolar stainless steel wire electrode. Electrically evoked responses were recorded using glass microelectrode filled with ACSF (3-5 M $)$ in the stratum radiatum of the rat hippocampus. At the beginning of the measurement, the stimulus intensity was reduced to obtain $30-50 \%$ of maximal excitatory postsynaptic potential (EPSP) at stimulus frequency of $0.033 \mathrm{~Hz}$ as the so-called baseline response. After 10-15 min of a stabilization period, long-term potentiation (LTP) of neuronal transmission was induced by a single train of HFS (100 Hz, 1 s). After an LTP induction, further evoked responses were recorded at baseline stimulus frequency $(0.033 \mathrm{~Hz})$ for the next $40 \mathrm{~min}$. Average baseline response recorded during $10 \mathrm{~min}$ before HFS was normalized to 1 , and consequently, all further responses were recalculated to normalized values.

\section{STATISTICAL EVALUATION}

The data were statistically evaluated using the InStat software version 2.05 (GraphPad) and GraphPad Prism Software (GraphPad, La Jolla, USA). Data were expressed as means \pm standard error of the mean (SEM). One-way analysis of variance (ANOVA) was used to evaluate (1) the difference among all the experimental groups (using the Tukey-Kramer multiple comparison test) and (2) the difference obtained compared to the control groups (using the Dunnett multiple comparison test). The limit of $p<0.05$ was considered a statistically significant difference.

\section{RESULTS}

In biochemical determination of the lipid profile, TG levels before the diet were elevated in HTG rats compared to the Wistar group, which is consistent with their genetic status. Diet and administration of SMe1EC2 did not affect the TG levels. In the atorvastatin-treated group, there was a decrease in TG levels compared to their pretreatment level (Fig. 1A). HFFD in combination with hypertriacylglycerolemia increased the levels of TC and LDL-cholesterol in blood serum compared to Wistar rats. The administration of atorvastatin and SMe1EC2 at a lower dose resulted in the reduction of the increase in the levels of TC (Fig. 1B) and LDL-cholesterol (Fig. 1C). The higher dose of SMe1EC2 did not affect the lipid profile of blood serum. HDL-cholesterol levels were significantly reduced by the use of HFFD in combination with hypertriacylglycerolemia. Treatment did not affect the HDLcholesterol (Fig. 1D). In electrophysiological measurements, HFFD in combination with hypertriacylglycerolemia deteriorate the course of LTP and administration of 

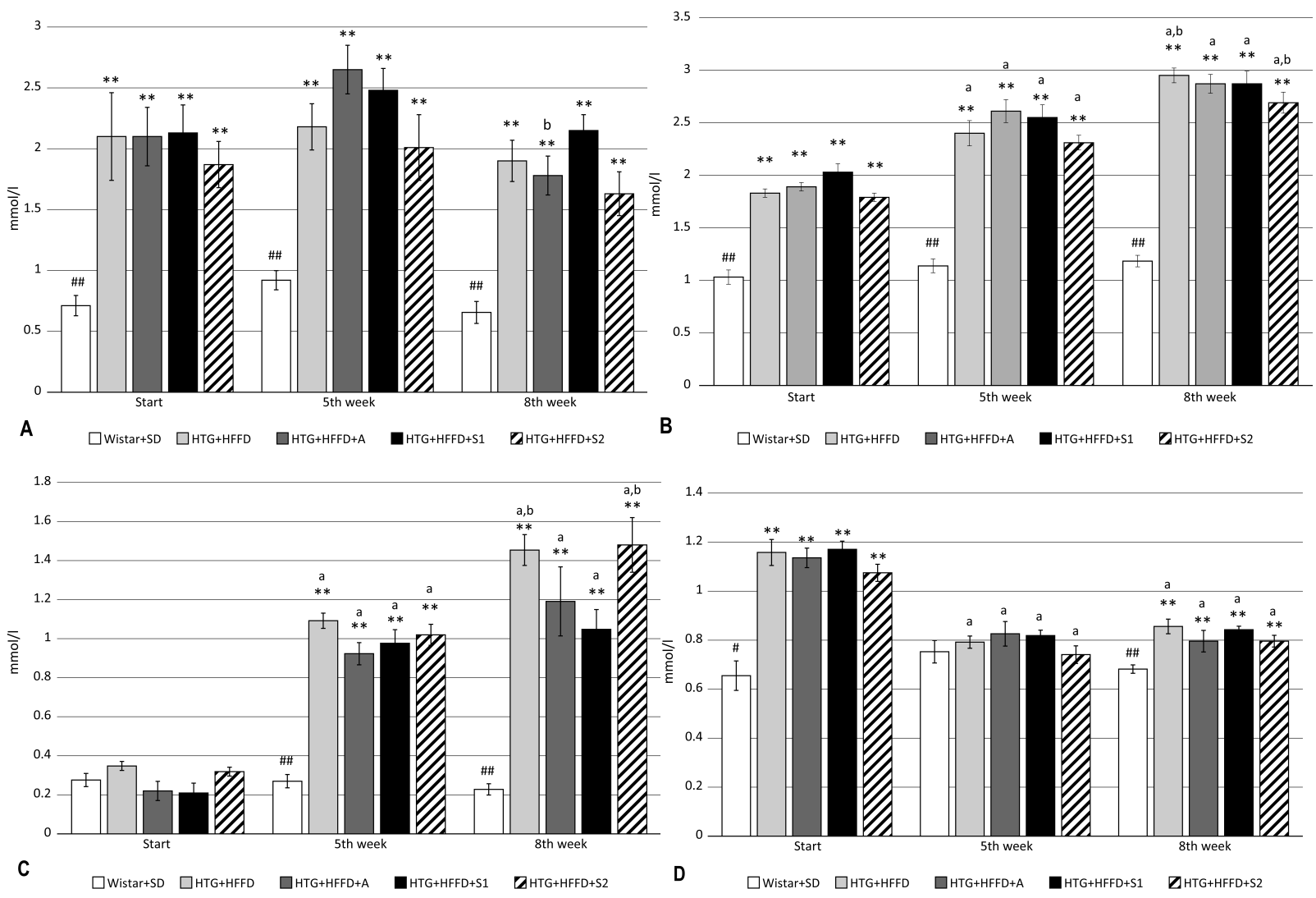

Figure 1. Lipid profile ( $\mathrm{mmol} / \mathrm{l})$ in blood serum during the 8-week diet. (A) Levels of triacylglycerols (TG) before the diet were elevated in hypertriacylglycerolemic (HTG) rats compared to Wistar group. High-fat-fructose diet (HFFD) and administration of SMe1EC2 did not affect the TG levels. Atorvastatin decreased TG levels compared to their pretreatment level. (B) HFFD in combination with hypertriacylglycerolemia increased the levels of total cholesterol (TC) in blood serum compared to Wistar rats. The administration of atorvastatin and SMe1EC2 at a lower dose resulted in the reduction of the increase in TC levels. The higher dose of SMe1EC2 did not affect the levels of TC in blood serum. (C) HFFD in combination with hypertriacylglycerolemia increased the levels of LDL-cholesterol in blood serum compared to Wistar rats. The administration of atorvastatin and SMe1EC2 at a lower dose resulted in the reduction of the increase in LDL-cholesterol levels. The higher dose of SMe1EC2 did not affect the levels of LDL-cholesterol in blood serum. (D) HDL-cholesterol levels were significantly reduced by the use of HFFD in combination with hypertriacylglycerolemia. Wistar-SD, the negative control group fed with standard diet; HTG-HFFD, the positive control group of HTG rats fed with HFFD; HTG+HFFD+A, HTG rats fed with HFFD and treated from the 6th to 8th week with atorvastatin $(25 \mathrm{mg} / \mathrm{kg}) ;$ HTG + HFFD+S1, HTG rats fed with HFFD and treated from the 6th to 8th week with SMe1EC2 with lower dosage $(0.5 \mathrm{mg} / \mathrm{kg}) ;$ HTG+HFFD+S2, HTG rats fed with HFFD and treated from the 6th to 8th week with SMe1EC2 at higher dosage $(25 \mathrm{mg} / \mathrm{kg})$. Each group consisted of 10 rats. The values on the chart are arithmetic means \pm SEM. The limit of $p<0.05$ was considered as a statistically significant difference. ${ }^{*}$ significant difference compared to Wistar (before diet, after 5 weeks of diet [before treatment], after 8 weeks of diet [after 3 weeks of treatment]); \# significant difference compared to the HTG-HFFD group (before diet, after 5 weeks of diet [before treatment], after 8 weeks of diet [after 3 weeks of treatment]). $a$, significant difference between values of the same group compared to values before diet; $b$, significant difference between values of the same group compared to values after 5 th week of diet.

atorvastatin and SMe1EC2 at both doses tested resulted in improvement in the course of LTP (Fig. 2).

\section{DISCUSSION}

MetS is a worldwide health problem that can lead to cardiovascular and cerebrovascular diseases. We studied the effect of HFFD on lipid profile of blood serum and neurotransmission in the hippocampus of HTG rats. The main feature of HTG rats are elevated TG levels that are present from 10 weeks of their age, which may further lead to the development of dyslipidemia characterized by decreased HDL-cholesterol levels and elevated LDLcholesterol levels (Štolba et al., 1992). The HFFD causes non-alcoholic liver steatosis (Chukijrungroat et al., 2017), insulin resistance, and increases in cholesterol levels in 


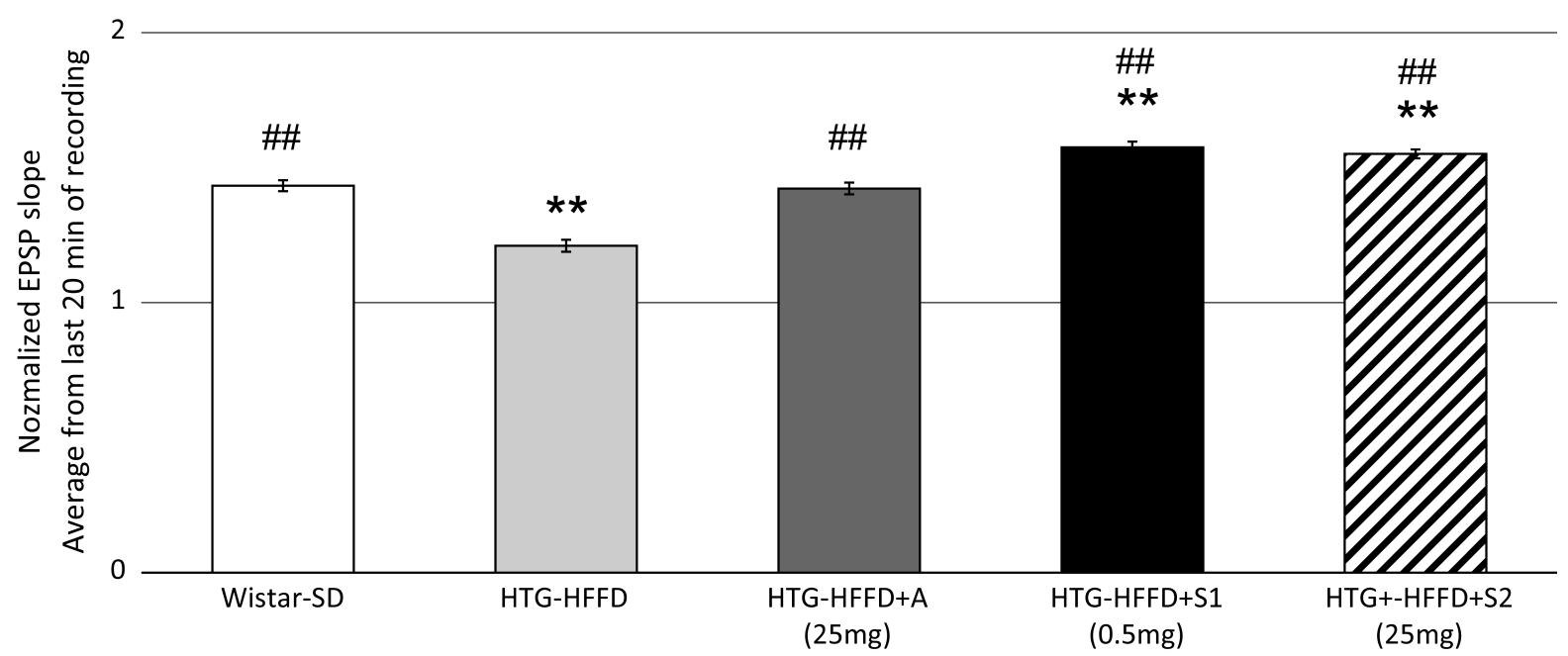

Figure 2. Electrophysiological measurement of long-term potentiation (LTP) in hippocampal stratum radiatum. High-fatfructose diet (HFFD) in hypertriacylglycerolemic (HTG) rats deteriorated the course of LTP and the administration of atorvastatin and SMe1EC2 at both doses tested resulted in an improvement in the course of LTP. Wistar-SD, the negative control group fed with the standard diet, $n=10$ hippocampal slices; HTG-HFFD, the positive control group of HTG rats fed with HFFD, $n=14$ hippocampal slices; HTG+HFFD+A, HTG rats fed with HFFD and treated from the 6th to 8th week with atorvastatin (25 mg/ $\mathrm{kg}), n=13$ hippocampal slices; HTG+HFFD+S1, HTG rats fed with HFFD and treated from the 6th to 8th week with SMe1EC2 at lower dosage $(0.5 \mathrm{mg} / \mathrm{kg}), n=14$ hippocampal slices; HTG+HFFD+S2, HTG rats fed with HFFD and treated from the 6th to 8th week with SMe $1 E C 2$ at higher dosage $(25 \mathrm{mg} / \mathrm{kg}), n=15$ hippocampal slices; each group consisted of 10 rats. The values on the chart are arithmetic means \pm SEM. The column chart shows arithmetic means \pm SEM of normalized EPSP slope recorded during last $20 \mathrm{~min}$. The limit of $p<0.05$ was considered as a statistically significant difference. ${ }^{*}$ Difference is significant compared to Wistar rats; \# difference is significant compared to the HTG-HFFD group.

blood plasma (de Sousa Rodrigues et al., 2017). Recently, it was reported that levels of TC, TG, and LDL-cholesterol were higher and levels of HDL-cholesterol were significantly reduced in Sprague-Dawley rats fed with HFD (Jia et al., 2013). Furthermore, rats of Fischer 344 strain fed with HFD had elevated levels of TC, TG, LDL-cholesterol, and even HDL-cholesterol after diet (Granholm et al., 2008). Krishna et al. (2015) found that HFD consumption altered synaptic plasticity, evidenced by significant reductions in LTP magnitude. Valladolid-Acebes et al. (2012) also published an attenuation of the LTP response in the radiatum layer in rats fed with HFD. In our work, we found that HFFD increased the levels of TC and LDL-cholesterol and decreased the levels of HDL-cholesterol in blood serum of HTG rats. Levels of TG before the diet were elevated in HTG rats compared to Wistar group, and the diet did not affect these values further. In electrophysiological measurement we found that HFFD diet deteriorated the course of LTP. We also investigated the effect of SMe1EC2 and atorvastatin treatment on lipid profile and hippocampal neurotransmission in HTG rats fed with HFFD. We chose the pyridoindole antioxidant SMe1EC2 because of its wide spectrum of protective action. According to previous studies conducted at the Slovak Academy of Sciences, SMe1EC2 is not toxic and has neuroprotective and cardioprotective effects (Horáková and Štolc, 1998). SMe1EC2 reduced the serum levels of TC and TG (Bezek et al., 2010). Its effect on plasma lipid levels is comparable to fenofibrate (Kysel'ová et al., 2010). The effect of SMe1EC2 was compared with the effects of atorvastatin, which is clinically used as lipid-lowering agent. Atorvastatin is an HMG-CoA reductase inhibitor effectively reducing LDL-cholesterol levels and TG levels and increasing HDL-cholesterol concentrations (Cornier et al., 2008). Atorvastatin acts as an immunomodulator by inhibiting the activation of T-lymphocytes. It reduces oxidative stress and improves endothelial function. Statins act as anti-inflammatory agents by several mechanisms (Meyer-Sabellek and Brasch 2006). In our study, SMe1EC2 at a lower dose and atorvastatin caused a reduction in the increase in the levels of TC and LDL-cholesterol and atorvastatin induced a decrease in the levels of TG, which is consistent with previous studies with HFD without fructose. Furthermore, SMe1EC2 at a lower dose improved the course of LTP at the rat hippocampus. This could be due to its neuroprotective as well as antioxidant effects. These results indicate that SMe1EC2 could be promising in the treatment of MetS-related disorders. This has, however, to be verified in further studies.

\section{ACKNOWLEDGMENTS}

The study was supported by the Slovak Grant Agency for Science VEGA 2/0054/15 (2015 -2018): Rizikové faktory 
kardiovaskulárnych a cerebrovaskulárnych ochorení a farmakologické možnosti ich ovplyvnenia/ Risk factors of cardiovascular and cerebrovascular diseases and pharmacological possibilities of their influence.

\section{ABBREVIATIONS}

ANOVA - Analysis of variance

HDL-cholesterol - high-density lipoprotein cholesterol
HFD - high-fat diet

HFFD - high-fat-fructose diet

HTG - hypertriacylglycerolemic

LDL-cholesterol - low-density lipoprotein cholesterol

LTP - long-term potentiation

MetS - metabolic syndrome

$n$ - number

TC - total cholesterol TG - triacylglycerols

\section{References}

[1] Alfaro F. J.: Lioutas V. A., Pimentel D. A., Chung C. C., Bedoya F., Yoo W. K., Novak V.: Cognitive decline in metabolic syndrome is linked to microstructural white matter abnormalities. J Neurol. 2016;263:2505 -2514.

[2] Bezek Š., Paulovičová E., Sotníková R., Navarová J., Gajdošík A, Bauer V., Dřímal J., Nosálová V., Kysel'ová Z.: The effect of novel pyridoindole derivative SMe1EC2 and atorvastatin on selected parameters in hereditary hypertriglyceridemic rats fed high-cholesterol diet. Presented at: International Symposium. Nitric Oxide: From Basic Regulation to Lifestylerelated Diseases; September 16-21, 2009; Tučepi, Croatia.

[3] Cornier M.A., Dabelea D., Hernandez T.L., Lindstrom R.C., Steig A.J., Stob N.R., Van Pelt R.E., Wang H., Eckel R.H.: The metabolic syndrome. Endocr Rev. 2008;29:777-822.

[4] Chukijrungroat N., Khamphaya T., Weerachayaphorn J., Songserm T., Saengsirisuwan V: Hepatic FGF21 mediates sex differences in high-fat high-fructose diet-induced fatty liver. Am J Physiol Endocrinol Metab. 2017;313:203-212.

[5] Granholm A., Bimonte-Nelson H. A., Moore A. B., Nelson M. E., Freeman L. R., Sambamurti K.: Effects of a saturated fat and high cholesterol diet on memory and hippocampal morphology in the middle -aged rat. J Alzheimer's Dis. 2008;14:133-145.

[6] Horáková L., Štolc S.: Antioxidant and pharmacodynamic effects of pyridoindole stobadine. Gen Pharmacol, 1998;30:627-638.

[7] Janczura M., Bochenek G., Nowobilski R., Dropinski J., Kotula-Horowitz K., Laskowicz B., Stanisz A., Lelakowski J., Domagala T.: The relationship of metabolic syndrome with stress, coronary heart disease and pulmonary function - An occupational cohort-based study. PloS One, 2015;10:1-20.

[8] Jia Y-J., Liu J., Guo Y-L., Xu R-X., Sun J., Li J-J.: dyslipidemia in rat fed with high-fat diet is not associated with PCSK9-LDLreceptor pathway but ageing. J Geriatr Cardiol. 2013;10:361368.

[9] Krishna S., Keralapura M. M., Lin Z., Wagner J. J., de La Serre C. B., Harn D. A., Filipov M. M.: Neurochemical and electrophysiological deficits in the ventral hippocampus and selective behavioral alterations caused by high-fat diet in female C57BL/6 mice. Neuroscience. 2015;297:170-181.

[10] Kysel'ová Z., Sotníková R., Navarová J., Gajdošík A., Paulovičová E., Bernátová I., Bezek Š.:The effect of SMe1EC2 and fenofibrate on selected parameters in hereditary hypertriglyceridemic rats fed high -cholesterol diet. Presented at: International
Symposium. Nitric Oxide: From Basic Regulation to Lifestylerelated Diseases; September 16-21, 2009; Tučepi, Croatia.

[11] Launer L. J., Masaki K., Petrovitch H., Foley D., Havlik R. J.: The association between midlife blood pressure levels and latelife cognitive function. JAMA. 1995;274:1846-1851.

[12] Liu Z., Patil I. Y., Jiang T., Sancheti H., Walsh J. P., Stiles B. L., Yin F., Cadenas E.: High -fat diet induces hepatic insulin resistance and impairment of synaptic plasticity. Am J Pathol. 2009;175: $355-364$.

[13] Meyer-Sabellek W., Brasch H.: Atherosclerosis, inflammation, leukocyte function and the effect of statins. J Hypertens, 2006;24:2349-2351.

[14] O’Neil S, O’Driscoll L.: Metabolic syndrome: a closer look at the growing epidemic and its associated pathologies. Obesity Reviews, 2015;16:1-12.

[15] de Sousa Rodrigues M. E., Bekhbat M., Houser M. C., Chang J., Walker D. I., Jones D. P., Oller do Nascimento C. M. P., Barnum Ch. J., Tansey M. G.: Chronic psychological stress and high -fat high fructose diet disrupt metabolic and inflammatory gene networks in the brain, liver, and gut and promote behavioral deficits in mice. Brain Behav Immun. 2017;59:158-172

[16] Štolba P., Dobešová Z., Hušek P., Opltová H., Zicha J., Vrána A., Kuneš J.: The hypertriglyceridemic rat as a genetic model of hypertension and diabetes. Life Sci. 1992;51:733-740.

[17]Treviño S., Vázquez-Roque R. A., López-López G., Perez-Cruz C., Moran C., Handal-Silva A., González-Vergara E., Guevara J., Díaz A.: Metabolic syndrome causes recognition memory impairments and reduced the hippocampal neuronal plasticity in rats. J Chem Neuroanat. 2017; 82:65 -75.

[18] Valladolid-Acebes I., Merino B., Principato A., Fole A., Barbas C., Lorenzo M. P., Garcia A., Del Olmo N., Ruiz-Gayo M., Cano V.: High-fat diets induce changes in hippocampal glutamate metabolism and neurotransmission. Am J Physiol Endocrinol Metab. 2013;302:396-402.

[19] Qiu C., Winblad B., Fratiglioni L.: The age-dependent relation of blood pressure to cognitive function and dementia. Lancet Neurol. 2005;4:8487-8499.

[20] Yates K. F., Sweat V., Yau P. L., Turchiano M. M., Convit A.: Impact of metabolic syndrome on cognition and Brain. Arterioscler Thromb Vasc Biol. 2012;32:2060-2067.

[21]Yau P. L., Castro M. G., Tagani A., Tsui W.H., Convit A.: Obesity and metabolic syndrome and structural brain impairments in adolescence. Pediatrics, 2012;130: 856-864. 